\title{
Loopedia, a Database for Loop Integrals
}

\section{Bogner}

Institut für Physik, Humboldt-Universität zu Berlin, D-10099 Berlin, Germany

\section{S. Borowka}

Theoretical Physics Department, CERN, Geneva, Switzerland*

\section{T. Hahn ${ }^{\dagger}$}

Max-Planck-Institut für Physik, Föhringer Ring 6, D-80805 Munich

E-mail: hahn@mpp.mpg • de

\section{G. Heinrich}

Max-Planck-Institut für Physik, Föhringer Ring 6, D-80805 Munich

\section{S.P. Jones}

Max-Planck-Institut für Physik, Föhringer Ring 6, D-80805 Munich

\section{Kerner}

Max-Planck-Institut für Physik, Föhringer Ring 6, D-80805 Munich

\section{A. von Manteuffel}

Dept. of Physics \& Astronomy, Michigan State University, East Lansing, MI 48824, USA

\section{Michel}

CP3, Université Catholique de Louvain, B-1348 Louvain-la-Neuve, Belgium

\section{E. Panzer}

All Souls College, University of Oxford, OX1 4AL, Oxford, UK

\section{Papara}

Max-Planck-Institut für Physik, Föhringer Ring 6, D-80805 Munich

Loopedia is a new database at loopedia.org for Feynman integrals, providing both bibliographic information as well as results made available by the community. Its bibliometry is complementary to that of INSPIRE or arXiv, as it allows to search for integrals by graph-theoretical specifications, e.g. their topology.

Loops and Legs in Quantum Field Theory (LL2018)

29 April 2018 - 04 May 2018

St. Goar, Germany

\footnotetext{
* former address

†Speaker.
} 


\section{Introduction}

Searching and accessing bibliographic information in high-energy physics is generally solved satisfactorily through databases such as INSPIRE and arXiv. The search within these databases is indexed by 'traditional' metrics: author, title, year of publication, etc. However, for the practitioner in loop calculations it is not easy to find e.g. publications which refer to a particular loop integral, where the integral is specified in some graph-theoretical way, say by its topology.

Loopedia [1] attempts to fill this gap, while not being limited to bibliographic information. The description field of each record can hold any kind of textual information (e.g. URLs to software), and in addition arbitrary files can be uploaded, for example Fortran programs or Maple worksheets.

\section{Integrals and Graphs}

Scalar Feynman loop integrals correspond to graphs and as such can be identified by a representation of the corresponding graph. A common choice is the edge (adjacency) list: each propagator (edge) is specified by the pair of vertices (nodes) it connects - external nodes need not be distinguished to identify the topology. For example, the following two-loop box graph has the edge list $(\mathrm{e}, 0)(0,1)(0,2)(\mathrm{e}, 1)(1,3)(2,3)(2,4)(3,5)(\mathrm{e}, 4)(4,5)(\mathrm{e}, 5)$ :

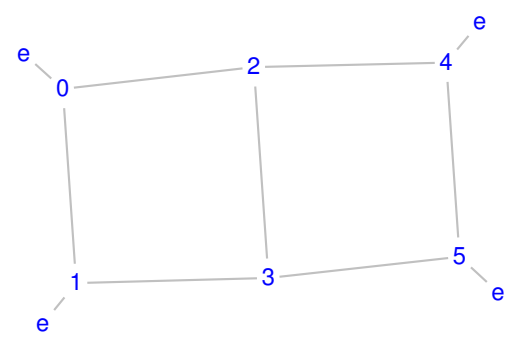

The Nickel index $[2,3,4]$ provides an alternative representation which is constructed as follows: start at a vertex 0 and write down all vertices connected to 0 (e for external legs), then insert $a^{\prime}$ ' $\mid$ '. Repeat the procedure for the other vertices but omit edges already included. For the two-loop box graph above this procedure leads to

$\begin{array}{lcccccc}\text { vertex } & 0 & 1 & 2 & 3 & 4 & 5 \\ \text { connected to } & e 12 & e[0] 3 & {[0] 34} & {[12] 5} & e[2] 5 & e[34]\end{array}$

and hence its Nickel index is e $12|e 3| 34|5| e 5|e|$.

The Nickel index provides a compact identifier and has therefore been chosen as the basic object by which integrals are indexed in Loopedia. Neither the edge list nor the Nickel index is unique, however, i.e. depending on the chosen labelling the same graph may have different edge lists/Nickel indices. Loopedia therefore canonicalizes all input.

Besides this 'bare' Nickel index, which represents just the topology of the graph, Loopedia introduces an augmented form, the colored Nickel index, CNickel for short, which additionally captures the configuration of masses and external $q^{2}$. Loopedia color-codes them on the screen: 


\section{Nickel e12|e3|34|5|e5|e| \\ CNickel e12|e3|34|5|e5|e|:110|10|00|0|11|1|}

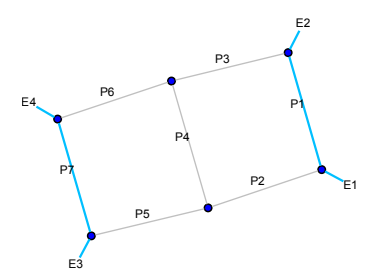

For each 'edge identifier' of the Nickel (e or a digit), the coloring has a mass identifier:

0 for zero,

$\{z, n, s\}$ for $\{$ any, non-zero, special $\}$ mass scale,

$1 \ldots 9$, a... $\mathrm{y}$ (not $\mathrm{n}, \mathrm{s}$ ) for a definite non-zero mass scale.

The 's' option is for cases like thresholds or pseudo-thresholds, and the exact meaning of 'special' should be detailed in the description of the integral.

The 'definite' identifiers also represent arbitrary scales (though not zero) but unlike the ' $z$ ns' choices they express equality of scales within the graph, similar to named patterns in a computeralgebra system. The graph in the example above has several massive propagators/ legs, but all with the same scale ' 1 '.

\section{User Guide}

Loopedia is located at loopedia.org and when accessed, the Start Page is displayed. One enters a graph in the input field and/or chooses constraints for the search with the lower control bars and then hits the Search button.

The graph is entered via its edge list. The user has some freedom there: generally the input is understood as long as a pairing of vertices into propagators can reasonably be inferred from it. This means that e.g. FeynArts [5] or QGRAF [6] notation can directly be pasted into the input form. An edge list is internally converted to a Nickel index right away. Alternatively, the Nickel index can also be entered directly.

\subsection{Browsing Graphs}

The search results are displayed in Loopedia's Graph Browser (Fig. 1). The matches are organized such that the topology appears on the left-hand side and the configurations for which information is available are on the right. Every graph icon is clickable.

Clicking on one of the graph icons opens the single-graph display for that graph. For icons in the left column it starts the Configuration Editor (Fig. 2), for icons in the right column it opens the Record Display (Fig. 3). The Graph Browser is skipped and the Configuration Editor is started immediately if the search delivers no matches and an explicit graph was entered, to commence adding a new record.

\subsection{Viewing Single Graphs}

The navigation panel at the top has buttons to edit the configuration Edit $\bullet:$, the topology Edit - , to browse the configurations of the topology Browse $\bullet: *$, and to return to the start page Home . Depending on the situation not all buttons may be active. 


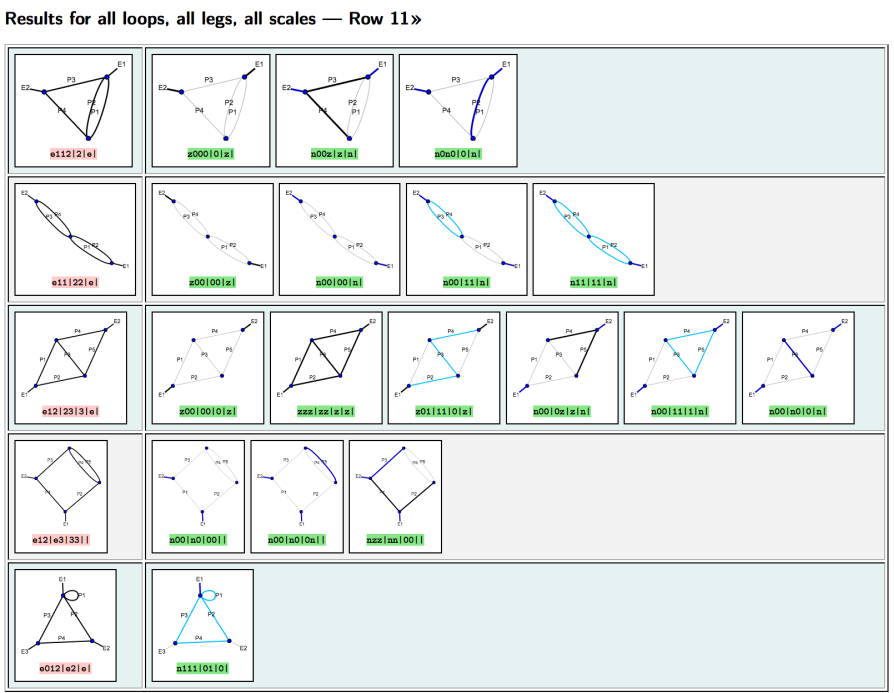

Prev Next Show 50 rows per page Home

Figure 1: The Loopedia Graph Browser
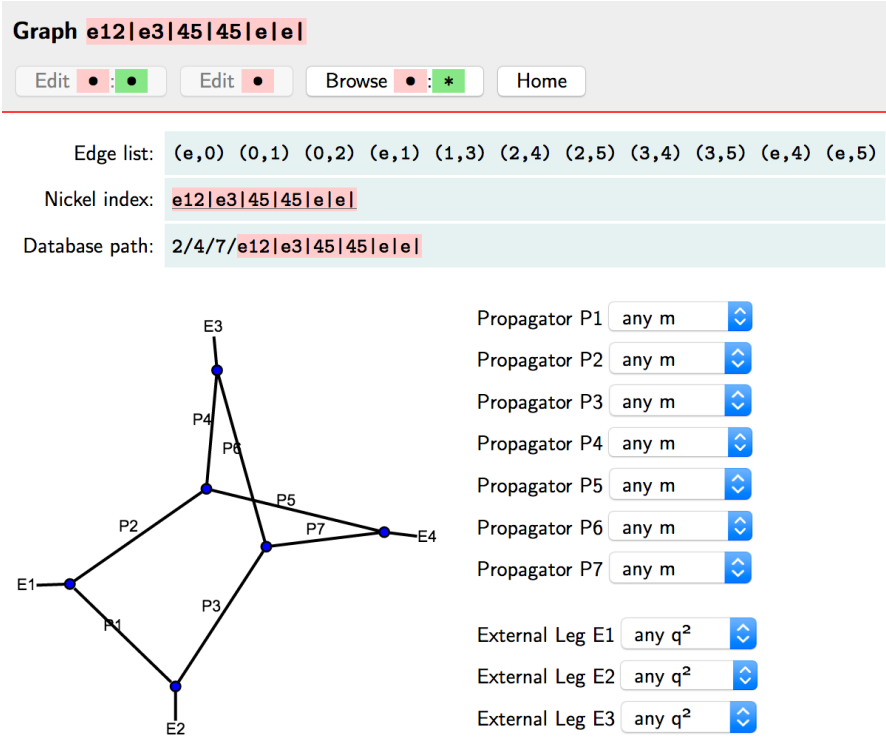

Propagator P1 any $m$
Propagator $P 2$
any $m$

Choose Configuration

Figure 2: The Loopedia Configuration Editor

In the main part of the page, a picture of the graph is shown underneath its various textual representations (edge list, [C]Nickel index, database path). To the right the Configuration Chooser is displayed, with the defaults preset to the current values (if any).

When editing a topology (bare Nickel index only) the page ends here, i.e. in order to proceed one needs to choose a configuration first. Otherwise the page continues with the available records and the New Record Form (Fig. 4). 


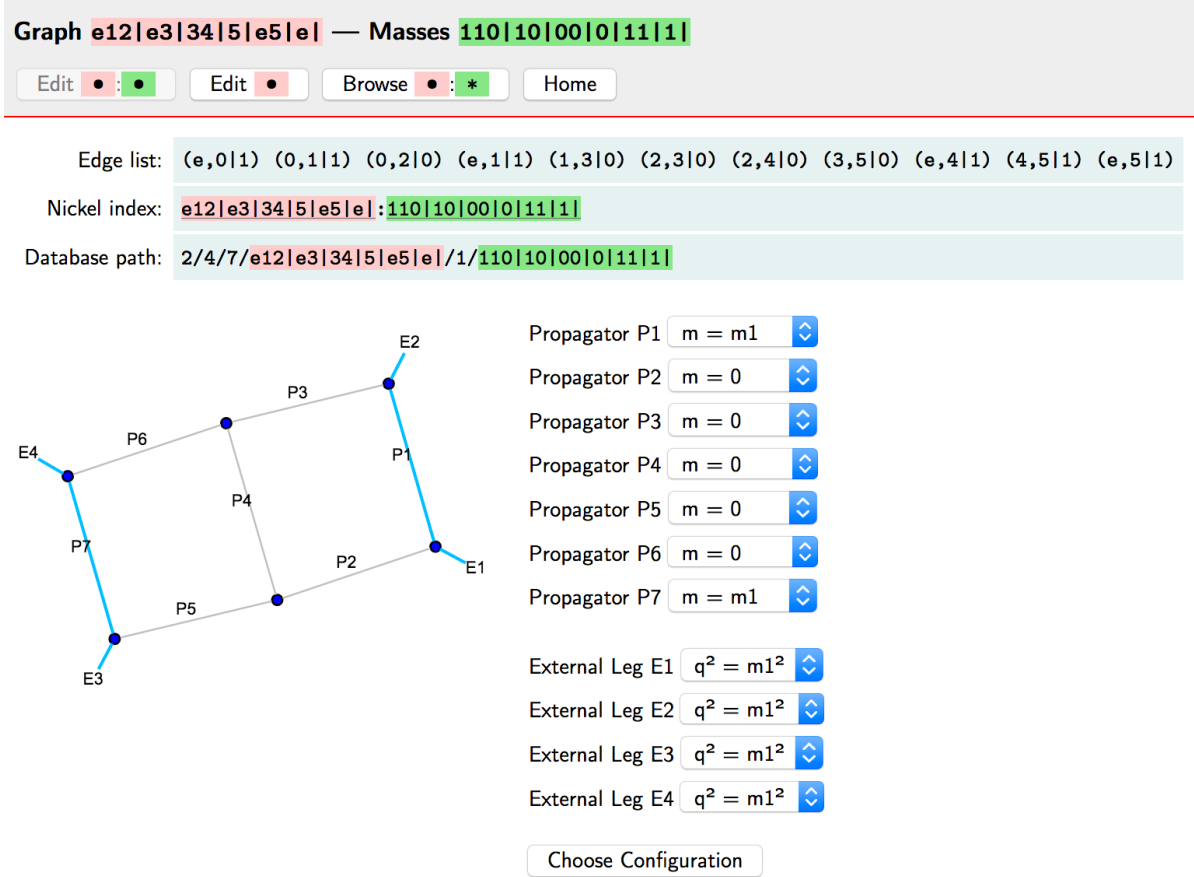

Figure 3: The Loopedia Record Display

\subsection{Entering New Records}

To enter a new record one must first navigate to a graph and a configuration as outlined above. The New Record Form is found beneath the available records.

Upon successful submission the submitted record is displayed again for review, with controls for editing and deletion (Fig. 5). An e-mail is sent to the submitter's address with an individualized URL that allows to access the review page again for future editing.

The submitted records are not visible publicly before they have been confirmed by a moderator. This is a safety measure we implemented currently to prevent situations like submitters deleting each other's records; this may change in the future. The moderators are notified together with the submitter and will usually make new records public in a timely manner.

\section{Internal structure}

Loopedia is realized as a bash script in a CGI environment which uses the Unix file system as database and is indexed by the mlocate utility.

The database contains (in the real filesystem sense) one directory for each bare Nickel index, with subdirectories for the configuration index (the second part of the CNickel) of the same graph. Underneath comes another layer of subdirectories for the visibility (public, private, deleted), and below that, one directory for each record. The actual implementation inserts a few more directory layers for indexing and performance reasons so that the full database path becomes 


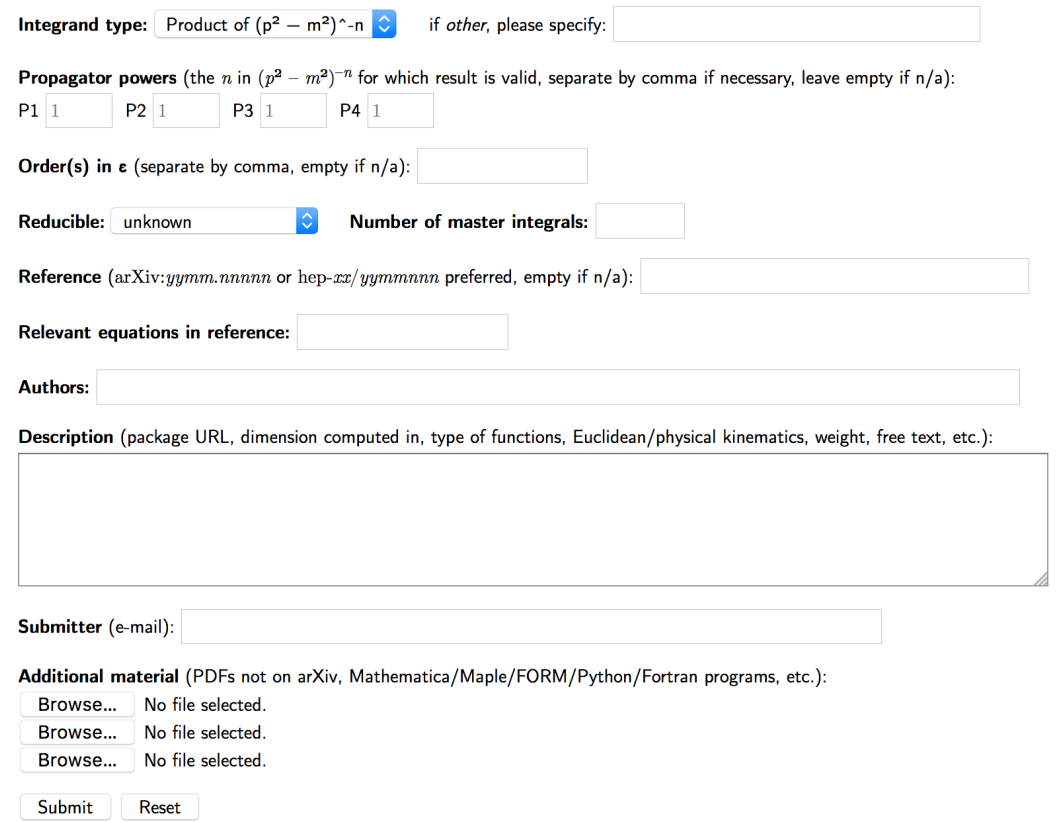

Figure 4: The New Record Form

\begin{tabular}{|l|r|}
\hline Reference: arXiv:1612.05609 & $\begin{array}{r}\text { Record 1482239373.Z1Fv } \\
\text { added 20 Dec 2016 13:09 UTC } \\
\text { Description: The authors compute the planar 2-loop box master integrals involved in QQ } \rightarrow \text { QQ, } \\
\text { where QQ are massive external quarks using the method of differential equations. } \\
\text { Submitter: } \underline{\text { sophia.borowka@cern.ch }}\end{array}$ \\
last modified 23 May 2017 14:07 UTC \\
edit delete
\end{tabular}

This record is not publicly visible until confirmed by a moderator.

Done

Figure 5: The Loopedia Record Review

where $L, \ell, p, s$ are the number of loops, legs, propagators, and scales, respectively. The database root ' $\mathrm{db}$ ' is included in the path to be able to make anchored searches. Records have identifiers similar to the maildir format in Unix, e.g. 1482239373 . Z1Fv.

Almost all graph-theoretical operations in Loopedia are taken care of by the Python library GraphState [4]. GraphState allows to supplement a graph with configurations, i.e. add extra integers to either the nodes or the edges. The colored Nickel index used by Loopedia implements a single edge coloring which encodes masses and external $q^{2}$.

To draw the graph, a Python function translates the graph into Graphviz's DOT language [7], from which neato produces an SVG image. SVG is an XML-based vector format rendered by all modern browsers and scales well in the icon-size plaquettes of the Graph Browser.

\section{Summary}

We presented a new database which allows to search for bibliographic and other information on loop integrals based on topology-related information. The intention of Loopedia is to collect the information available in the literature and to make it searchable by graph. It relies on the concept 
that the community will actively contribute to the database by submitting new integral information. The database is hosted at the Max Planck Institute for Physics in Munich with broadband internet access and a daily backup at loopedia.org.

\section{References}

[1] C. Bogner et al., "Loopedia, a Database for Loop Integrals," arXiv:1709.01266 [hep-ph].

[2] J.F. Nagle, J. Math. Phys. 7 (1966) 1588.

[3] B.G. Nickel, D.I. Meiron, G.A. Baker, Compilation of 2-pt and 4-pt graphs for continuous spin model, University of Guelph report, 1977.

[4] D. Batkovich, Y. Kirienko, M. Kompaniets, S. Novikov, arXiv:1409.8227. The GraphState library is at https://pypi.python.org/pypi/GraphState.

[5] J. Küblbeck, M. Böhm, A. Denner, Comput. Phys. Commun. 60 (1990) 165; T. Hahn, Comput. Phys. Commun. 140 (2001) 418 [hep-ph/0012260].

[6] P. Nogueira, J. Comput. Phys. 105 (1993) 279

[7] E.R. Gansner, S.C. North, Software - Practice and Experience 30 (2000) 1203, graphvi z . org. 